\title{
The in vitro effect of leukocyte- and platelet-rich fibrin (L-PRF) and cross- linked hyaluronic acid on fibroblast viability and proliferation.
}

SADJ July 2018, Vol 73 no 6 p395 - p399

MT Peck ${ }^{1}$, D Hiss ${ }^{2}$, L Stephen ${ }^{3}$, A Olivier. ${ }^{4}$

\begin{abstract}
Introduction: Leukocyte- and platelet-rich fibrin (L-PRF), an autologous derived platelet and leukocyte concentrate, was first introduced by Choukroun et al. in 2001 and is currently used for several oral and maxillofacial procedures. Hyaluronic acid $(\mathrm{HA})$ is an anionic glycosaminoglycan that shows a wide range of physiological actions, acting as a cellular scaffold, stimulating cell adhesion and migration, as well as having anti-inflammatory effects.
\end{abstract}

Aims and objectives: To investigate the effect of crosslinked $\mathrm{HA}$ and L-PRF on fibroblast viability and proliferation.

Methods: An in vitro laboratory study was conducted at The University of the Western Cape. L-PRF was prepared from a single healthy volunteer and cross-linked HA was obtained from a commercially available product. Analysis was carried out on 3T3 cells using MTT assay.

Results: Both L-PRF and cross-linked HA are associated with cell viability and increased cell proliferation. L-PRF had it strongest proliferative effect after 24 hours whereas $\mathrm{HA}$ had it strongest effect after eight days $(p<0.05)$. The combination of HA and L-PRF was not significantly better than the control $(p>0.05)$.

Conclusions: L-PRF prepared in a specific manner as outlined, as well as commercially available cross-linked $\mathrm{HA}$, is compatible with cell growth and proliferation.

1. Mogammad Thabit Peck: BChD, MSc, MChD, MRD Edin, FDS RCSSEd. Department of Oral Medicine and Periodontology, University of the Western Cape.

2. Donavon Hiss: MSc(Med), PhD(Medicine). Department of Medical Biosciences, University of the Western Cape.

3. Lawrence Stephen: $B C h D, P h D$. Department of Oral Medicine and Periodontology, University of the Western Cape.

4. Annette Olivier: BSC, Hons B, M(Phys Ed). Oral and Dental Research Department, University of the Western Cape.

\section{Corresponding author}

Mogammad Thabit Peck:

Department of Oral Medicine \& Periodontology, Faculty of Dentistry,

University of the Western Cape, P/Bag X1, Tygerberg 7505, South Africa.

Tel: 021937 3186. Fax: 086618 7560. E-mail: mpeck@uwc.ac.za.

\section{ACRONYMS}

ЗТЗ cells: mouse fibroblast cell line

bFGF: basic fibroblast growth factor

CIHA: cross-linked Hyaluronic acid

DMEM: Dulbecco's Modified Eagle's Medium

EDTA: ethylene-diamine-tetra-acetic acid

EGF: epidermal growth factor

FBS: foetal bovine serum

HA: $\quad$ hyaluronic acid

HGF: human gingival fibroblasts

L-PRF: leukocyte- and platelet-rich fibrin

PDGF-AB: platelet-derived growth factor

TGF-ק1: transforming growth factor beta 1

VEGF: vascular endothelial growth factor

\section{NTRODUCTION}

Leukocyte- and platelet-rich fibrin (L-PRF), an autologous derived platelet and leukocyte concentrate, was first introduced by Choukroun et al. in 2001 and is currently used for a number of oral and maxillofacial procedures. The use of L-PRF has gained popularity in the field of surgical implantology, specifically for procedures involving soft and hard tissue augmentation. ${ }^{2-5}$ The preparation of L-PRF differs from those of previous platelet concentrates in that it involves the use of a single spin protocol that results in an easily manipulated biomaterial that can be applied directly to the site of surgery. ${ }^{1}$ It is assumed that the concentrated growth factors associated with L-PRF optimize wound healing and decrease surgical recovery time. ${ }^{2,5}$ Consequently, L-PRF has been used in various fields, including dermatology and periodontal regeneration. ${ }^{2-5}$

Hyaluronic acid $(\mathrm{HA})$ is an anionic, glycosaminoglycan. ${ }^{6,7}$ It is a naturally occurring molecule that is found in high concentrations in the extracellular matrix of skin, cartilage,

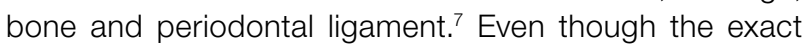
function of HA has not yet been established, the molecule shows a wide range of physiological actions, acting as a 
cellular scaffold, stimulating cell adhesion and migration, as well as having anti-inflammatory effects. ${ }^{7}$ Commercially synthesized HA, in various forms, has been used in tissue engineering, dermatology, orthopaedics, and more recently, periodontal regeneration. . $^{7-10}$ Traditionally, two forms of commercially synthesized HA exist, i.e., non-cross-linked $\mathrm{HA}$, and cross-linked-HA, resulting in different properties and indications for each form of $\mathrm{HA}$.

The aim of the study was to investigate the effect of cross-linked HA and L-PRF on fibroblast viability and proliferation.

\section{MATERIALS AND METHODS}

The study was conducted at The University of the Western Cape, Cape Town, South Africa, in 2017. Ethical clearance was obtained from the Research Ethics Committee of the above university (reference number: BM 16/3/31). Informed consent was obtained from the blood donor.

\section{Preparation of the L-PRF}

Thirty six milliliters of blood was obtained from a single 56 year old healthy female volunteer. The blood samples were collected in blood collecting tubes that contained clot activator i.e., Vacuette ${ }^{\circ}$ 9-ml serum tubes with Z Serum Clot Activator (Greiner BioOne International AG, Germany). These were then immediately centrifuged at 400xg for 12 minutes in a standard benchtop centrifuge (PLC03, Hicare International, Taiwan) as has been previously described..$^{11}$ Centrifugation separated the blood into three distinct layers (Figure 1). The layers could be distinguished as a topmost layer consisting of platelet poor plasma, L-PRF in the middle, and red blood cells below. L-PRF was then removed with a sterile forceps, separated from the underlying red blood cells and shredded into smaller fragments using surgical scissors.

Hyaluronic Acid (HA)

A commercially available cross-linked HA (hyaDent BG, Bioscience $\mathrm{GmbH}$ ) was used in the present study. It is sold as a clear gel contained in a dental anaesthetictype cartridge and is applied using a dental syringe and a specifically supplied needle. This specific formulation contains $2.0 \mathrm{mg} / \mathrm{ml}$ of sodium hyaluronate that is crosslinked with butanediol digylcidyl ether, resulting in a HA molecule with a unique chemical structure.

Cells and Cell Culture

In this investigation, the 3T3 fibroblast cell line was obtained from The National Repository for Biological

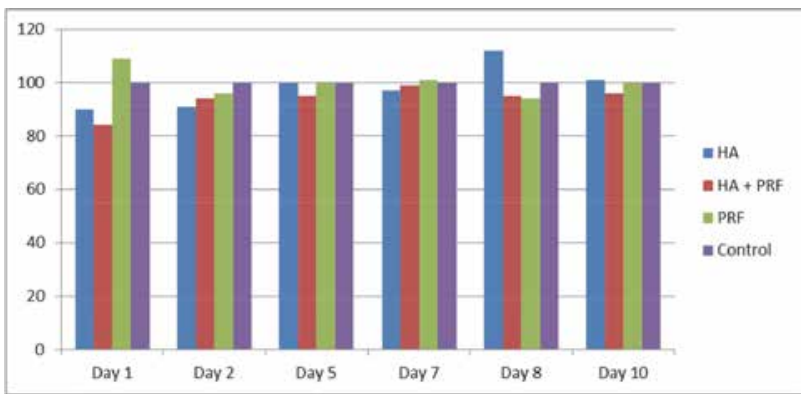

Figure 1: Proliferation and viability assay (MTT) of 3 T3 cells. Control is taken as $100 \%$
Materials (Sandringham, South Africa). The 3T3 cells were incubated at $37^{\circ} \mathrm{C}$ in $5 \%$ carbon dioxide and $95 \%$ humidity in Dulbecco's Modified Eagle's medium (DMEM) with 10\% foetal bovine serum (FBS) and 1\% penicillin- streptomycin mix. Cells were grown to about $80 \%$ confluence and then trypsinized using trypsin-ethylene-diamine-tetra-acetic acid (EDTA). The cells were then seeded into $3 \times 96$-well plates and treated as follows;

- Plate 1 - 1 drop of HA (HA group)

- Plate 2-1 drop of HA added to a $1 \mathrm{~mm} \times 1 \mathrm{~mm}$ fragment of L-PRF (HA + L-PRF group)

- Plate 3-1 mm x $1 \mathrm{~mm}$ fragment of L-PRF (L-PRF group)

All the plates had an equal number of controls containing only DMEM. After a culture period of 24 hours to 10 days, each group was removed and proliferation and viability evaluated using the 3-(4,5-dimethylthiazol-2-yl)-2,5diphenyltetrazolium bromide (MTT) assay. ${ }^{12}$ A minimum of five replicate experiments for each group was performed to ensure reproducibility.

\section{Cell Viability and Proliferation Assay}

Cell viability (the metabolic activity of the cell) and cell proliferation (rate of cell division) were measured using the standard MTT assay (MTT, Sigma Chemical Co., Baltimore, MD, USA). The MTT assay or test is a sensitive and quantitative colorimetric assay that measures these parameters. ${ }^{12}$ It reflects the capacity of the mitochondria in living cells to convert MTT to purple formazan crystals that can be solubilized with dimethylsulphoxide (DMSO) or isopropanol. ${ }^{12}$ This effect is directly proportional to the cell number and is measured with a spectrophotometer at a wavelength of $540 \mathrm{~nm}$. Rapidly dividing cells i.e. cells which show high rates of proliferation, reduce MTT at a faster rate than cells with low proliferative potential. The MTT assay therefore measures the ability of the cell to reduce MTT (viability) as well as the rate of cell division (proliferation). As a result, the MTT assay can also be used to measure cytotoxicity (loss of viable cells) and cytostatic activity (shift from proliferation to quiescence). For the current study, readings were taken from all groups with the control group set at $100 \%$.

\section{Data Analysis}

Data from the MTT assay were captured using Microsoft Excel 2010 (Microsoft Corporation, Washington, USA) and statistically analyzed using the one-way ANOVA and Tukey's test.



Figure 2: HA vs control (MTT assay). 


\section{RESULTS}

With the control group set at $100 \%$, the mean optical densities of each of the test groups were divided by that of the control group and expressed as a percentage of the control value. The data from the MTT assay was correlated, and is presented in Figures 1-4. Statistical analysis is represented by Tables 1 and 2 .

\begin{tabular}{|c|c|c|c|c|c|c|c|}
\hline & \multicolumn{2}{|c|}{ HA } & \multicolumn{2}{|c|}{$\mathrm{HA}+\mathrm{PRF}$} & \multicolumn{2}{|c|}{ PRF } & \multirow[b]{2}{*}{$P$ value } \\
\hline & Mean & SD & Mean & SD & Mean & SD & \\
\hline Day 1 & 89.98 & 9.83 & 85.44 & 12.35 & 108.99 & 5.43 & 0.005 * \\
\hline Day 2 & 91.41 & 8.79 & 94.72 & 9.89 & 96.27 & 4.34 & 0.632 \\
\hline Day 5 & 100.08 & 8.79 & 95.18 & 7.29 & 101.01 & 7.26 & 0.47 \\
\hline Day 7 & 97.47 & 8.92 & 98.6 & 6.15 & 100.79 & 5.77 & 0.757 \\
\hline Day 8 & 112.2 & 9.69 & 94.57 & 4.09 & 94.78 & 9.66 & $0.007^{*}$ \\
\hline Day 10 & 100.88 & 6.46 & 96.15 & 2.62 & 99.15 & 7.64 & 0.439 \\
\hline \multicolumn{8}{|c|}{$\left({ }^{*} p<0.05\right)$} \\
\hline
\end{tabular}

\section{Table 2: Comparison between the test groups (Tukey test)}

\begin{tabular}{|l|c|c|c|}
\hline & HA vs Control & HA-PRF vs Control & PRF vs Control \\
\hline Day 1 & $0.02^{*}$ & $0.01^{*}$ & $0.003^{*}$ \\
\hline Day 2 & $0.03^{*}$ & 0.13 & $0.045^{\star}$ \\
\hline Day 5 & 0.49 & 0.08 & 0.38 \\
\hline Day 7 & 0.27 & 0.31 & 0.08 \\
\hline Day 8 & $0.01^{*}$ & $0.009^{*}$ & 0.13 \\
\hline Day 10 & 0.38 & $0.005^{\star}$ & 0.49 \\
\hline ( $\mathrm{P}<0.05)$ & & & \\
\hline
\end{tabular}

According to the results obtained, both L-PRF and cross-linked $\mathrm{HA}(\mathrm{clHA})$ as well as the combination of the two materials were compatible with cell viability and proliferation (i.e. none of the tested biomaterials tested displayed evidence of being cytotoxic or cytostatic). Crosslinked HA showed a statistically significant reduction in cell number when compared with the control group at 24 hours (89.98\% $\pm 9.83, p<0.05)$. Thereafter cell viability steadily increased, reaching $100.88 \% \pm 6.46$ at day 10 . Peak cell proliferation associated with HA was seen on day 8 and recorded as $112.20 \% \pm 9.69$. This was significantly greater than the proliferation seen in both other test groups for the same time period $(p<0.05)$. The HA + L-PRF group showed a cell viability of $85.44 \% \pm 12.35$ at 24 hours. This was statistically significant $(p<0.05)$ when compared with

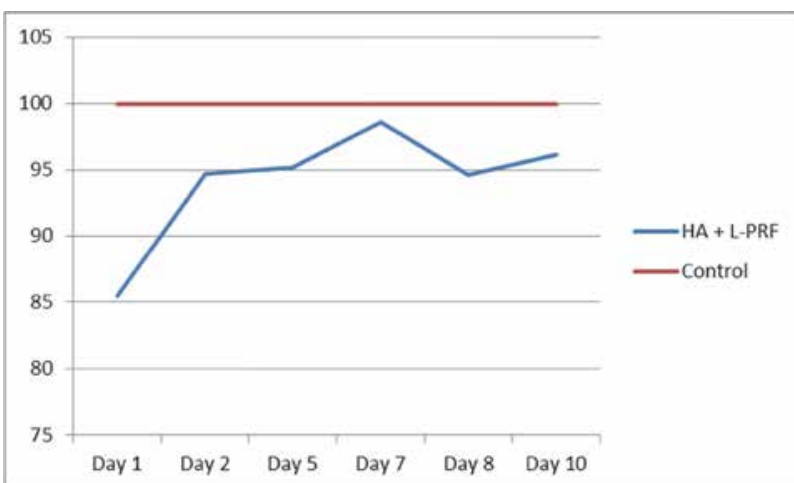

Figure 3: HA + L-PRF vs Control (MTT assay). the control group, indicating a negative effect on cellular proliferation in the first 24 hours. This difference was not significant for days 2, 5 and 7, but was again recorded on days 8 and $10(p<0.05)$.

The L-PRF group showed the highest cell viability and proliferation after 24 hours $(108.99 \% \pm 5.43)$. This was significantly higher than the other groups tested as well as higher than the control group ( $p<0.05)$. At day 10, no significant differences were seen between all the test groups, however the HA + L-PRF group showed a significantly reduced number of cells as compared with the control $(p<0.05)$.

A comparison of the proliferative effects of the test groups indicate that both groups containing clHA showed a pattern of cellular proliferation that steadily increased from day 1 to peak at day 8 (clHA group) and day 7 (HA+L-PRF group) respectively. This was different to that observed for the L-PRF group where peak cell proliferation was seen after 24 hours and steadily decreased over the test period (Figure 4.)

\section{DISCUSSION AND CONCLUSIONS}

The results from the current study indicate that both L-PRF and cross-linked HA are biocompatible and induce cellular proliferation of fibroblasts in vitro.

\section{L-PRF}

It appears as if the effect of L-PRF used in this study is most pronounced in the first 24 hours when compared with any of the other materials tested. This may be due to the high concentration of growth factors present in the material. These include substances such as vascular endothelial growth factor (VEGF), basic fibroblast growth factor (bFGF), plateletderived growth factor (PDGF-AB), transforming growth factor beta 1 (TGF- $\beta 1$ ) and epidermal growth factor (EGF), released from platelets as well as leukocytes. ${ }^{13-16}$ This initial "proliferative burst" is similar to that seen by Vahabi et al. who reported a statistically significant increase in cellular proliferation of human gingival fibroblasts (HGFs) after 24 hours. ${ }^{17}$ However, unlike the Vahabi et al. study, which showed a reduction of cell viability of up to $60 \%$ after 72 hours, the present study showed sustained cellular viability and proliferation that was statistically similar to the control group for all subsequent test days. The difference in observations in the two studies is difficult to explain without a direct comparison between

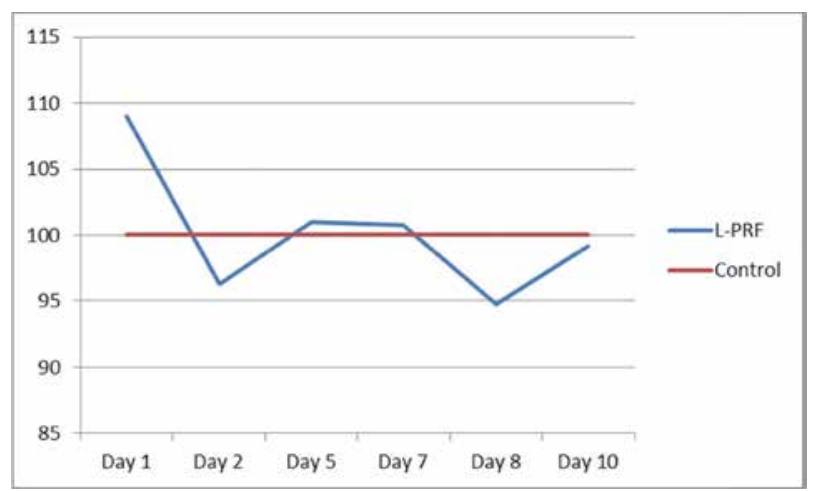

Figure 4: L-PRF vs control (MTT assay). 
the two biomaterials associated with each investigation. Dohan Ehrenfest et al. showed that no significant differences were seen in cell viability and proliferation when measured using the MTT assay over 21 days for HGFs cultured with L-PRF 13. However, the MTT assay was only reported after day three, thereby possibly failing to record any earlier significant findings. Similar to the Dohan Ehrenfest et al investigation, the present study showed no statistically significant differences when L-PRF was compared to the control group at day 7 , and for subsequent days thereafter.13 A recent study investigating the in vitro effect of a modified protocol to produce L-PRF, also showed no significant cell proliferation after 24 hours compared with the control group, suggesting that the protocol used to produce L-PRF in the current study may result in a L-PRF with a different biological effect compared with the abovementioned reports. ${ }^{16} \mathrm{~A}$ previous investigation by Peck et al. showed that the L-PRF used in this study has a distinct fibrin architecture with most of the fibres being of a "larger" diameter. ${ }^{18}$ Whether this characteristic is significant in the biological behaviour of the biomaterial is still unclear.

\section{Cross-linked HA}

A limited number of studies have reported on the cell stimulating potential of the specific high molecular weight $\mathrm{ClHA}$ used in the present study. ${ }^{6,7}$ Previous research indicates that this formulation is biocompatible and is able to positively stimulate the proliferation of periodontal ligament cells in vitro. ${ }^{6}$ These results were statistically significant when the biomaterial was diluted at ratios of 1:100 and 1:10. The authors can provide no conceivable reason why the diluted material evoked a higher proliferation rate than the undiluted material. We speculate, however, that this may possibly be due to the reduced viscosity of the material after dilution, thereby allowing better interaction with the growth medium and cell culture.

In the present study we showed that undiluted $\mathrm{clHA}$ resulted in a reduction of cellular proliferation for the first few days after cell culture. This was similar to what was observed by Fujioko- Kubayashee et al and colleagues. At day 5, cellular proliferation approximated that of the control group, and, by day 8 , was significantly higher than that of the control group $(p<0.05)$. Therefore an augmented proliferative effect was noticed for the present study. Although HA has well-established water retention properties, the exact mechanism of HA and fibroblast interaction is unclear with "fibroblast stretching" cited as the most likely cause. ${ }^{19}$ In a recent study evaluating the effect of both ClHA and non-cross linked HA on dermal fibroblasts, it was shown that the molecular structure, particularly the type and density of the cross-linkages, play a significant role in the ability of HA to stimulate fibroblast proliferation. ${ }^{19}$ Any biological effect of synthesized HA is product dependent and the results of each study should therefore be interpreted with this in mind. This might also explain the variations in clinical efficacy of dental related HA that are reported in the literature.

Hyaluronic Acid and L-PRF

Because HA has previously been used for tissue engineering and is commercially available for the management of several inflammatory-related conditions, the authors evaluated the cell viability and proliferative potential of a combination of ClHA and L-PRF on fibroblasts. As L-PRF has an inherently high concentration of growth factors related to wound healing, it was assumed that the combination of the two biomaterials would stimulate cellular proliferation. The results from the current study indicate that the combined formulation was biocompatible, but had a varied effect on cell proliferation. Similar to $\mathrm{ClHA}$, the $\mathrm{ClHA}+\mathrm{L}-\mathrm{PRF}$ mixture showed a significant reduction in cell proliferation after 24 hours. Proliferation then increased over time, and on day 7 , it peaked, showing results similar to those of the control group $(p>0.05)$. This proliferation was opposite to that seen for the L-PRF group. It is interesting to note that the clHA+L-PRF combination showed consistently lower cell proliferation when compared with the control group throughout the time period of the study. Although L-PRF on its own stimulated cell proliferation significantly after 24 hours, it seems the addition of clHA constrained this initial stimulatory effect. Whether this is related to the viscosity of the $\mathrm{ClHA}$ or its concentration is critical to debate. It is known that diluted forms of $\mathrm{clHA}$ seem to better stimulate cell growth, and this might therefore explain the results seen in the present study. ${ }^{6}$

\section{CONCLUSION}

L-PRF and ClHA are unique biomaterials that are being used in tissue engineering. In the present study, we show that these materials on their own, or in combination, are biocompatible and stimulate cell growth. Interestingly, it appears that this specific method of L-PRF preparation results in a material that has a stimulatory effect that peaks within the first 24 hours. Cross-linked HA also stimulates growth positively, but unlike L-PRF this effect is prolonged over 8 days, implying a different, as yet unknown, mechanism of action. The combination of L-PRF and ClHA seems to provide no further advantage to cellular growth when compared with using either of the materials on their own. Based on the above, we can speculate that although L-PRF results in an almost immediate stimulatory effect, that $\mathrm{ClHA}$, as a sole treatment choice, might be beneficial in the management of wounds that require more prolonged stimulation. Further research is required to determine the clinical implications of the above findings.

\section{References}

1. Choukroun J, Adda F, Schoeffler C, Vervelle A. Une opportunité en paro-implantologie: Le PRF. Implantodontie 2001; 42; 55-62.

2. Nanditha S, Chandrasekaran B, Muthusamy S, Muthu K. Apprising the diverse facets of Platelet rich fibrin in surgery through a systematic review. Int J Surg. 2017;46:186-194.

3. De Pascale MR, Sommese L, Casamassimi A, Napoli C. Platelet derivatives in regenerative medicine: an update. Transfus Med Rev. 2015;29(1):52-61.

4. Martínez CE, Smith PC, Palma Alvarado VA. The influence of platelet-derived products on angiogenesis and tissue repair: a concise update. Front Physiol. 2015;206:290-7.

5. Verma UP, Yadav RK, Dixit M, Gupta A. Platelet-rich Fibrin: A paradigm in periodontal therapy - a systematic review. J Int Soc Prev Community Dent. 2017;7(5):227-33.

6. Fujioka-Kobayashi M, Müller HD, Mueller A, Lussi A, Sculean A, Schmidlin PR, Miron RJ. In vitro effects of hyaluronic acid on human periodontal ligament cells. BMC Oral Health. 2017;17(1):44-50 
7. Mueller A, Fujioka-Kobayashi M, Mueller HD, Lussi A, Sculean A, Schmidlin PR, Miron RJ. Effect of hyaluronic acid on morphological changes to dentin surfaces and subsequent effect on periodontal ligament cell survival, attachment, and spreading. Clin Oral Investig. 2017;21(4):1013-9.

8. Casale M, Moffa A, Vella P, Sabatino L, Capuano F, Salvinelli B, Lopez MA, Carinci F, Salvinelli F. Hyaluronic acid: Perspectives in dentistry. A systematic review. Int J Immunopathol Pharmacol. 2016;29(4):572-82.

9. Bertl K, Bruckmann C, Isberg PE, Klinge B, Gotfredsen K, Stavropoulos A. Hyaluronan in non-surgical and surgical periodontal therapy: a systematic review. J Clin Periodontol. $2015 ; 42(3): 236-46$

10. Bansal J, Kedige SD, Anand S. Hyaluronic acid: a promising mediator for periodontal regeneration. Indian J Dent Res. $2010 ; 21(4): 575-8$.

11. Peck MT, Marnewick J, Stephen L. Alveolar ridge preservation using leukocyte and platelet-rich fibrin: a report of a case. Case Reports in Dentistry 2011; 2011; 345048.

12. Mosmann T. Rapid colorimetric assay for cellular growth and survival: application to proliferation and cytotoxicity assays. J Immunol Methods. 1983;65(1-2):55-63.

13. Dohan Ehrenfest DM, Diss A, Odin G, Doglioli P, Hippolyte MP, Charrier JB. In vitro effects of Choukroun's PRF (platelet-rich fibrin) on human gingival fibroblasts, dermal prekeratinocytes, preadipocytes, and maxillofacial osteoblasts in primary cultures. Oral Surg Oral Med Oral Pathol Oral Radiol Endod. $2009 ; 108(3): 341-52$.

14. Clipet F, Tricot S, Alno N, Massot M, Solhi H, Cathelineau G, Perez F, De Mello G, Pellen-Mussi P. In vitro effects of Choukroun's platelet-rich fibrin conditioned medium on three different cell lines implicated in dental implantology. Implant Dent. $2012 ; 21(1): 51-6$.

15. Chisini LA, Karam SA, Noronha TG, Sartori LRM, San Martin AS, Demarco FF, Conde MCM. Platelet-poor plasma as a supplement for fibroblasts cultured in platelet-rich fibrin. Acta Stomatol Croat. 2017 ;51(2):133-40.

16. Fujioka-Kobayashi M, Miron RJ, Hernandez M, Kandalam U, Zhang Y, Choukroun J. Optimized platelet-rich fibrin with the low-speed concept: growth factor release, biocompatibility, and cellular response. J Periodontol. 2017 ;88(1):112-21.

17. Vahabi S, Vaziri S, Torshabi M, Rezaei Esfahrood Z. Effects of plasma rich in growth factors and platelet-rich fibrin on proliferation and viability of human gingival fibroblasts. J Dent (Tehran). $2015 ; 12(7): 504-12$.

18. Peck MT, Hiss D, Stephen LXG. The effect of preparation method on fibrin diameter of leukocyte- and platelet-rich fibrin (L-PRF). Submitted for publication. South African Dental Journal. 2017

19. Landau M, Fagien S. Science of hyaluronic acid beyond filling: fibroblasts and their response to the extracellular matrix. Plast Reconstr Surg. 2015 ;136(5 Suppl):188S-195S 